\title{
DIFFERENTIAL EQUATIONS FOR AUTOMORPHIC FORMS IN SEVERAL COMPLEX VARIABLES
}

\author{
H. L. RESNIKOFF
}

In a paper published in 1889 , Hurwitz [3] proved that the holomorphic automorphic forms and the meromorphic automorphic functions associated with groups arising from the Riemann surfaces of algebraic functions satisfy algebraic differential equations of order at most three; cf. [5], [8], and [11]. ${ }^{1}$ More precisely, given such a form or function $f$, there is a polynomial $P_{f} \in C\left[X_{0}, X_{1}, X_{2}, X_{3}\right]$ which is zero for the specialization $\left(X_{0}, X_{1}, X_{2}, X_{3}\right) \rightarrow\left(f, f^{\prime}, f^{\prime \prime}, f^{\prime \prime \prime}\right)$.

To generalize this theorem, let $Z$ be analytically equivalent to an irreducible bounded symmetric domain in the sense of $\hat{E}$. Cartan, and suppose that the (real) dimension of the Shilov boundary of $Z$ is equal to $\operatorname{dim}_{C} Z$, the complex dimension of $Z$. Suppose further that $\Gamma$ is a discrete group of holomorphic automorphisms of $Z$ which is pseudoconcave or such that $Z / \Gamma$ has a compactification as a connected complex space whose meromorphic functions extend all of the meromorphic functions on $Z / \Gamma$. The main result of this paper is the following:

THEOREM. If $\operatorname{dim}_{C} Z>1$, then every holomorphic $\Gamma$-automorphic form and every meromorphic $\Gamma$-automorphic function on $Z$ satisfies an algebraic differential equation of order at most equal to $\left(\operatorname{dim}_{C} Z+\operatorname{rank} Z\right)$.

The idea of the proof is simple. The hypotheses on $\Gamma$ insure that the Siegel-Thimm-Remmert-Andreotti-Grauert Theorem (cf. [14], [15], [10], and [1]) can be applied. It states that analytically dependent meromorphic functions on $Z / \Gamma$ are algebraically dependent. Therefore, given a holomorphic $\Gamma$-automorphic form $f$, the theorem will be proved if it is possible to construct $\left(1+\operatorname{dim}_{C} Z\right)$ additional $\Gamma$-automorphic forms which are polynomials in $f$ and its derivatives.

Polynomial differential operators that map forms to forms, and functions to functions, for all groups of holomorphic automorphisms of $Z$ will be constructed so that the individual characteristics of distinct discrete groups will not require separate analysis. The geometrical restrictions imposed on $Z$ make such a construction possible by using Jordan algebras [2], [6].

Received by the editors February 3, 1969.

' I am indebted to Professor H. Petersson for bringing Hurwitz' work to my attention. 
Let $\mathfrak{A}$ denote a simple compact real Jordan algebra. Then $Z(\mathfrak{A})$ $=\mathfrak{A}+i \exp \mathfrak{A}$ is a tube domain which is holomorphically equivalent to an irreducible bounded symmetric domain. $\mathfrak{A}$ is dense in the Shilov boundary of $Z(\mathfrak{H})$, so the dimension of the Shilov boundary $=\operatorname{dim} \mathfrak{A}$ $=\operatorname{dim}_{C} Z(\mathfrak{A})$. Conversely, Theorem 4.9 and [7, Remark 1, p. 286] show that every irreducible bounded symmetric domain whose complex dimension is equal to the real dimension of its Shilov boundary is holomorphically equivalent to some $Z(\mathfrak{A})$.

The full group $G(\mathfrak{A})$ of holomorphic automorphisms of $Z(\mathfrak{A})$ is generated by the real translations $z \rightarrow z+a$ with $z \in Z$ and $a \in \mathfrak{A}$, the involution $z \rightarrow-z^{-1}$, where the inverse is given by the Jordan structure, and by certain automorphisms of $\mathfrak{A}$ if $G(\mathfrak{H})$ is not connected. If $\Gamma \subset G(\mathfrak{A})$ and $f$ is a holomorphic automorphic form, then for each $\gamma \in \Gamma$,

$$
f(\gamma z)=\operatorname{det}\left(\frac{\partial \gamma z}{\partial z}\right)^{-k / 2 q} f(z)
$$

where $\partial \gamma z / \partial z$ denotes the Jacobian linear transformation,

$$
q=\operatorname{dim}_{C} Z(\mathfrak{A}) / \operatorname{rank} Z(\mathfrak{A})=\operatorname{dim}_{R} \mathfrak{A} / \text { rank } \mathfrak{A},
$$

and $k$ is an integer called the weight of the form $f ; \operatorname{det}(\partial \gamma z / \partial z)^{-1 / 2 q}$ is an irreducible polynomial.

Let $(\Gamma, k)$ denote the complex vector space of holomorphic $\Gamma$-automorphic forms of weight $k$. In order to define a polynomial differential operator with domain $(\Gamma, k)$ and range a subset of $\left(\Gamma, k^{\prime}\right)$ for some $k^{\prime}$, introduce the reduced trace $\sigma(a)$ of $a \in \mathfrak{A} . \sigma(a, b)=\sigma(a b)$ is a nonsingular bilinear form which extends to the complexification $\mathfrak{A}(i)$ of $\mathfrak{A}$. Let $\nabla_{z}$ denote the gradient differential operator with respect to $\sigma$ defined by

$$
\frac{\partial f}{\partial z}(a)=\sigma\left(\nabla_{z} f, a\right)
$$

for differentiable functions defined on open subsets of $\mathfrak{A}(i)$.

Four types of polynomial differential operators can be constructed from $\nabla$ in a natural way.

If $a \in \mathfrak{A}(i)$, let the reduced norm of $a$ be denoted by $|a|$; it is a homogeneous polynomial of degree equal to rank $\mathfrak{A}$ in the coordinates of $a$ with respect to a basis for $\mathfrak{A}$. The mapping $f \rightarrow|\nabla f|$ defines a nonlinear first order operator.

LEMMa 1. If $f$ is a meromorphic $\Gamma$-automorphic function, then $|\nabla f|$ is a meromorphic function on $Z(\mathfrak{A})$ which satisfies the functional equation of a $\Gamma$-automorphic form of weight 2. 
Proof. The Jacobian of a real translation is the identity, so that $|\nabla F|$ behaves correctly with respect to real translations. A straightforward calculation shows that $\nabla_{-z^{-1}}=P(z) \nabla_{z}$ where $P(z)$ is the "quadratic representation" of $\mathfrak{A} ;$ hence $\left|\nabla_{-z^{-1}} f\right|=|z|{ }^{2}\left|\nabla_{z} f\right|$. If $A$ is an automorphism of $\mathfrak{A}$, then $\left|\nabla_{\mathbf{A} z}\right|=\left|\nabla_{\mathfrak{z}}\right|$ since $\operatorname{det} A=1$, which completes the proof.

This differential operator converts automorphic functions to meromorphic automorphic forms, but it can also be used to map pairs of holomorphic automorphic forms to holomorphic automorphic forms. Suppose that $f \in(\Gamma, k)$ and $g \in(\Gamma, l)$, and set $r=\operatorname{rank} \mathfrak{A}$. Then $f^{l} / g^{k}$ is a $\Gamma$-automorphic function. It follows that

$$
E(f, g)=|l g \nabla f-k f \nabla g|=\left(g^{r(k+1)} / f^{r(l-1)}\right)\left|\nabla\left(f^{l} / g^{k}\right)\right|
$$

belongs to $(\Gamma, r(k+l)+2)$. This can be stated formally as

Lemma 2. $E:(\Gamma, k) \times(\Gamma, l) \rightarrow(\Gamma, r(k+l)+2)$ for all $\Gamma \subset G(Z(\mathfrak{U}))$.

Observe that $E$ is a first order nonlinear operator, and $E(f, f)=0$; it generalizes an operator used by Rankin [9].

The set $\exp \mathfrak{A}$ has a natural structure as a symmetric homogeneous space $G / K$. Let $d \mu$ be a conveniently normalized $G$-invariant measure on $\exp \mathfrak{A}$, and define the Laplace transform of an integrable function $f$ with domain $\exp \mathfrak{A}$ by

$$
\tilde{f}(p)=\int_{\exp \boldsymbol{g}} e^{-\sigma(p y)} f(y) d \mu
$$

where $p \in \operatorname{cl}(\exp \mathfrak{U})$. The mapping $f \rightarrow f^{*}$ defined by $f^{*}(y)=(-) r|y| f(y)$ induces a mapping of the Laplace transforms,

$$
f(p) \stackrel{\partial}{\rightarrow} \tilde{f}^{*}(p),
$$

which is a linear differential operator. $\partial$ was explicitly computed in [13]. Denote the $n$-fold composition of $\partial$ with itself by $\partial^{n}$.

LEMMA 3. (a) $\partial_{z}=\left|\nabla_{z}\right|$.

(b) $\partial^{n}:(\Gamma, q-n) \rightarrow(\Gamma, q+n)$ for all $\Gamma \subset G(Z(\mathfrak{X}))$.

The proof of this lemma appears in [13]. Part (b) is due to Selberg (unpublished).

If $q \neq n$, set

$$
D^{n} f=f^{(n-q) / k+n r \partial^{n} f^{(q-n) / k} .}
$$

It is easy to see that $D^{n} f$ is a polynomial in $f$ and its derivatives of order at most $n r$, and, as was shown in [12], 
Lemma 4. $D^{n}:(\Gamma, k) \rightarrow(\Gamma, n(k r+2))$ for all $\Gamma \subset G(Z(\mathfrak{U}))$.

REMARK. It is easily checked that

$$
|\nabla f|^{n}=\lim _{k \rightarrow 0} \prod_{j=0}^{n r-1}\left(\frac{q-n}{k}-j\right)^{-1} D^{n} f .
$$

Proof of theorem. Suppose that $f \in(\Gamma, k)$. Since $\operatorname{dim}_{C} Z>1, D^{1}$ exists and is of order rank $\mathfrak{A}$. Inductively define $E^{1} f=E\left(f, D^{1} f\right)$, $E^{n} f=E\left(f, E^{n-1} f\right) . E^{n}$ is a polynomial differential operator of order $(n+\operatorname{rank} \mathfrak{U})$. Let the weight of $E^{n} f$ be $n(f)$. Then (recalling that $r=\operatorname{rank} \mathfrak{A})$

$$
\left(D^{1} f\right)^{k} / f^{k r+2}, \quad\left(E^{1} f\right)^{k} / f^{1(\zeta)}, \cdots,\left(E^{\operatorname{dim} C Z} f\right){ }^{k} / f^{\operatorname{dim} C Z(f)}
$$

induce $\operatorname{dim}_{C} Z+1$ meromorphic functions on $Z / \Gamma$ which are, by the Siegel-Thimm-Remmert-Andreotti-Grauert Theorem, algebraically dependent. Clearing the denominator completes the proof for forms.

If $f$ is a meromorphic $\Gamma$-automorphic function, then $|\nabla f|$ is a meromorphic form of weight 2 . Hence

$$
|\nabla f|, \quad D^{1}|\nabla f|, \quad E^{1}|\nabla f|, \cdots, E^{\operatorname{dim} C Z-1}|\nabla f|
$$

are $\operatorname{dim}_{C} Z+1$ meromorphic forms which lead, just as above, to $\operatorname{dim}_{C} Z$ meromorphic functions. Together with $f$ itself, these are algebraically dependent. The order of $E^{\mathrm{dim} C Z-1}|\nabla f|$ is $\left(\operatorname{dim}_{C} Z-1\right)$ $+\operatorname{rank} Z+1=\operatorname{dim}_{C} Z+\operatorname{rank} Z$. Hence the theorem is proved.

REMARK. If $\operatorname{dim}_{C} Z=1$, then $D^{1}$ is not defined. The operator of least order mapping forms to forms is $D^{2}$ (see [8] and [11] for a proof). In this case, $\left\{f, D^{2} f, D^{3} f\right\}$ lead to differential equations for forms, and $\left\{f,|\nabla f|, D^{2}|\nabla f|\right\}$ for functions. These are Hurwitz' results.

\section{REFERENCES}

1. A. Andreotti and H. Grauert, Algebraische Körper von automorphen Funktionen, Nachr. Akad. Wiss. Göttingen Math.-Phys. Kl. II 1961, 39-48. MR 24 \#A2057.

2. H. Braun and M. Koecher, Jordan-Algebren, Springer-Verlag, Berlin, 1966. MR 34 \#310.

3. A. Hurwitz, "Utber die Differentialgleichungen dritter Ordnung welchen die Formen mit linearen Transformationen in sich genügen," in Mathemstische Werke. Band I, Basel, 1932, pp. 287-294; Cf. Math. Ann. 33 (1889), 345-352.

4. J. I. Igusa, On Siegel modular forms of genus two, Amer. J. Math. 84 (1962), 175200. MR $25 \# 5040$.

5. C. G. J. Jacobi, "Utber die Differentialgleichung, welcher die Reihen $1 \pm 2 q+2 q^{4}$

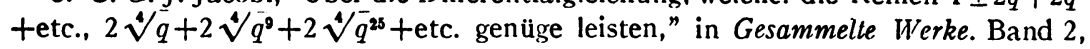
Springer-Verlag, Berlin, pp. 171-190; Cf. J. Reine Angew. Math. 36 (1848), 97-112. 
6. M. Koecher, Jordan algebras and their applications, Lecture notes, University of Minnesota, Minneapolis, Minn., 1962.

7. J. A. Wolf and A. Korányi, Realization of hermitian symmetric spaces as generalized half-planes, Ann. of Math. (2) 81 (1965), 265-288. MR $30 \# 4980$.

8. R. A. Rankin, The construction of automorphic forms from the derivatives of a given form, J. Indian Math. Soc. 20 (1956), 103-116. MR 18, 571.

9. - The construction of automorphic forms from the derivatives of given forms, Michigan Math. J. 4 (1957), 181-186. MR 19, 1172.

10. R. Remmert, Meromorphe Funktionen in kompakten komplexen Räumen, Math. Ann. 132 (1956), 277-288. MR 19, 171.

11. H. L. Resnikoff, On differential operators and automorphic forms, Trans. Amer. Math. Soc. 124 (1966), 334-346. MR 34 \#4490.

12. - Non trivial cusp forms in several complex variables, Proc. Conf. Complex Analysis, Rice Univ. Studies 54 (1968), 55-61.

13. $\longrightarrow$, On singular automorphic forms in several complex variables, Amer. J. Math. (to appear)

14. C. L. Siegel, Meromorphe Funktionen auf kompakten analytischen Mannigfaltigkeiten, Nachr. Akad. Wiss. Göttingen Math.-Phys. Kl. IIa 1955, 71-77. MR 17, 530.

15. W. Thimm, Uber die algebraischen Relationen zwischen meromorphen Funktionen in abgeschlossenen Räumen, Dissertation, Konigsberg, 1939.

RICE UNIVERSITY 